\title{
A MATHEMATICAL MODEL OF BONE REMODELING CONSIDERING MECHANOREGULATORY MECHANISMS: THEORETICAL MODEL DEVELOPMENT AND NUMERICAL STUDIES
}

\author{
S. Scheiner ${ }^{1, \dagger}$, P. Pivonka ${ }^{2}$, C. Hellmich ${ }^{1}$, D.W. Smith ${ }^{2}$ \\ ${ }^{1}$ Institute for Mechanics of Materials and Structures, Vienna University of Technology, Vi- \\ enna, Austria \\ ${ }^{2}$ Faculty of Engineering, Mathematics and Computing, The University of Western Australia, \\ Perth, Australia \\ $\dagger$ corresponding author: stefan.scheiner@tuwien.ac.at
}

\begin{abstract}
Bone remodeling involves the coordinated removal of bone by osteoclasts and addition of bone by osteoblasts, a process that is modulated by the prevailing mechanical environment. In this paper a fully coupled model of bone remodeling is developed, based on coupling a bone cell population model with a micromechanical homogenization scheme of bone stiffness. While the former model considers biochemical regulatory mechanisms between bone cells such as the RANK-RANKL-OPG pathway and action of TGF-beta, the latter model allows for accurate upscaling of the mechanical properties of bone. Importantly, we consider bone remodeling as being controlled proportionally to the microscopic strain energy density, on the observation scale where the sensing of the mechanical loading takes place, estimated by means of continuum micromechanics-based strain concentration. This approach allows to address two fundamental questions of bone biology: (i) How do biochemical changes influence bone remodeling and so affect the composition and mechanical properties of bone? and (ii) What mechanisms are responsible for mechanoregulation of bone remodeling? Numerical studies highlight the conceptual advantage of this new approach compared to conventional phenomenological models. It is demonstrated that the proposed model is able to simulate changes of the bone constituent volume fractions that are in qualitative agreement with experimental observations for osteoporotic and disuse syndromes.
\end{abstract}

Keywords: Bone remodeling, Mathematical modeling, Bone cell population dynamics, Continuum micromechanics, Mechanoregulation.

\section{INTRODUCTION}

Bone remodeling comprises the concerted activities of bone-resorbing osteoclasts and bone-forming osteoblasts, see [1, 2] for comprehensive reviews. Formulation of mathematical models, which include different cell types and regulatory factors in the description of bone 
remodeling, is challenging and only recently the research community has realized the need for such models in order to make links with clinical research. The first model which has included the action of biochemical factors on bone cells was formulated by [3]. This model has been revisited by $[4,5]$, who refined and extended the considered biochemical factors,

and evaluated the resulting bone cell populations in terms of the temporally changing bone volume. While the latter model gives valuable insights regarding implications of biochemical changes caused by diseases and/or therapeutic treatments on the populations of involved bone cells, no information is provided on how the mechanical integrity of bone is influenced under such circumstances. This deficit is the motivation for the first question addressed in this paper:

\section{How do biochemical changes affect the composition and the mechanical properties of} bone?

Experiments show that, apart from the prevailing biochemical conditions, mechanical loads acting upon bone also significantly influence the activation and sequence of bone remodeling-related mechanisms $[6,7,8,9]$. The sensing of the local mechanical environment and the transduction of the sensed signal to corresponding cellular events is managed by a third bone cell type, osteocytes [10], differentiating from osteoblasts which have been entrapped during bone formation in the composite of collagen fibres and hyroxyapatite crystals, commonly referred to as extracellular bone matrix. Thereby, osteocytes populate the lacunar pores within the extracellular bone matrix. The porous material consisting of extracellular material and lacunae is standardly called extravascular matrix. The exact functioning of mechanosensing in the course of bone remodeling remains to be unraveled, but experimental results strongly support the hypothesis that the deformation of the extravascular bone matrix (hosting osteocytes which reside in lacunae) is a major indicator for the exertion of influence on bone remodeling through mechanical forces [11]. This hypothesis is the starting point for tackling the second question of this paper:

II. What are the mechanism giving rise to mechanical regulation of bone remodeling and how can they be captured by a mathematical model?

Given the importance of the bone matrix deformation for answering this question, knowledge of the actual bone stiffness is crucial. Predictive modeling of the mechanical properties of bone requires consideration of its distinctive hierarchical organization [12,13]. Continuum micromechanics $[14,15]$ has proven to be well suited for this task, providing reliable estimates of bone stiffness $[16,17,18]$ based on the volume fractions of the bone constituents discernible on the investigated observation scales (from wet collagen to cortical bone), on the universal mechanical properties of the basic building blocks of bone (collagen, hydroxyapatite, and water), and on the mechanical interactions between bone constituents. Provided that both universal mechanical properties and interactions are invariant over the lifetime of bone $[19,17]$, the volume fractions remain as the key sources accountable for bone stiffness changes over time, e.g. in the course of diseases [20] and aging of bone [21], but also due to long-ranging alteration of the mechanical loading exerted onto bone [7]. However, stateof-the-art micromechanical models do not consider the bone constituent volume fractions as 
being controlled by the underlying bone cell activities. Rectifying this shortcoming directly relates to the answer to question I.

In summary, this paper is devoted to development of a fully coupled model of bone remodeling, based on a bone cell population model and a micromechanical homogenization scheme of bone stiffness, which contributes to answering the research questions raised above. This new approach allows for both biochemical and biomechanical regulation of bone remodeling, and will be implemented based on extension of the previous bone cell population model (BCPM) by introduction of mechanoregulatory mechanisms; application of the revised BCPM to a representative volume element of cortical bone, composed of extravascular bone matrix and Haversian canals, and analysis of the resulting cell populations in terms of corresponding bone constituent volume fractions; using the volume fractions as input for a micromechanical, one-step homogenization scheme which provides the macroscopic stiffness tensor of cortical bone; and development of a new model for mechanosensing and the corresponding modulation of bone remodeling, based on the microscopic strain energy density, rather than on the commonly considered macroscopic one. The capabilities of this methodology are then elucidated and discussed in the course of numerical studies.

\section{A bio-chemo-mechanically coupled model of bone remodeling}

\subsection{Fundamental mechanisms}

Bone remodeling is the continuous process of bone resorption and bone formation. Bone cells involved in bone remodeling derive from two different cell lines. Osteoblastic cells (responsible for bone formation) originate from mesenchymal stem cells, whereas osteoclastic cells (responsible for bone resorption) originate from hematopoietic stem cells. It is well known that bone remodeling is regulated by both biochemical and biomechanical mechanisms, which we will briefly sketch subsequently - for details see $[4,5]$ and references therein.

By binding to respective cell receptors, transforming growth factor- $\beta$ (TGF- $\beta$ ) promotes differentiation of uncommitted osteoblast progenitor cells into osteoblast precursor cells, whereas it inhibits differentiation of osteoblast precursor cells into active osteoblasts $[22,23]$. Furthermore, TGF- $\beta$ promotes osteoclast apoptosis. The RANK-RANKL-OPG signaling pathway [24] comprises expression of the receptor activator of nuclear factor kappa $\beta$ (RANK) on the surface of hematopoietic stem cells, and release of osteoprotegerin (OPG) by osteoblasts. Binding of RANKL (the ligand of RANK, expressed on osteoblast precursor cells) to RANK (expressed on osteoclast precursor cells) has been identified as key mechanism for promoting differentiation of osteoclast precursor cells into active osteoclasts. The decoy receptor molecule OPG (produced primarily by active osteoblasts) competes with RANK to bind to RANKL, and thus inhibits osteoclast differentiation. Many systemic hormones are known to affect bone remodeling. We consider only parathyroid hormone (PTH) in our model, due to its crucial importance for RANK-RANKL-OPG system - it increases the production of RANKL and reduces the production of OPG.

Furthermore, bone cells do not only respond to biochemical regulatory factors, but also 
to mechanical stimuli. The response of osteoclasts and osteoblasts to the applied mechanical loading is mediated by a third type of bone cells, osteocytes, which are able to effectively sense and transduce mechanical stimuli [10]. We assume that osteocytes are homogeneously distributed across the extravascular bone matrix and thus equally experience the mechanical environment to which the investigated volume of extravascular bone matrix is subjected. Experiments suggest [25] that catabolic mechanoregulation, resulting from disuse (i.e. the mechanical loading is lower than "normal"), leads to upregulation of osteoclast differentiation and thus to increased bone resorption. On the other hand, anabolic mechanoregulation, resulting from overuse (the mechanical loading is higher than "normal"), leads to increase proliferation of osteoblasts and thus increased bone formation. Disuse-related mechanoregulation is considered through a mechanically controlled term of RANKL-production, whereas overuse-related mechanoregulation is implemented using mechanically controlled proliferation of osteoblast precursor cells.

\subsection{Governing equations}

This section is devoted to briefly presenting the mathematical model governing the mechanisms described in the previous section and the interfaces between systems biology and continuum micromechanics-based stiffness homogenization.

\subsubsection{Bone cell dynamics}

Essentially, our model is based on the notion of a representative volume element (RVE). This implies that within an RVE bone remodeling together with the underlying (average) biochemistry and the mechanical environment is assumed to be uniform. Hence, spatial effects can be neglected and the first core module of our model, a bone cell population model (BCPM), providing the temporal evolutions of the concentrations of osteoblast precursor cells $\left(C_{\mathrm{OB}_{\mathrm{p}}}\right)$, active osteoclasts $\left(C_{\mathrm{OC}_{\mathrm{a}}}\right)$ and active osteoblasts $\left(C_{\mathrm{OB}_{\mathrm{a}}}\right)$ during ongoing bone remodeling, comprises a set of ordinary differential equations:

$$
\begin{aligned}
& \frac{\mathrm{d} C_{\mathrm{OB}_{\mathrm{p}}}}{\mathrm{d} t}=\mathscr{D}_{\mathrm{OB}_{\mathrm{u}}} C_{\mathrm{OB}_{\mathrm{u}}} \pi_{\mathrm{a}, \mathrm{T}}+\mathscr{P}_{\mathrm{OB}_{\mathrm{p}}} C_{\mathrm{OB}_{\mathrm{p}}} \Pi_{\varepsilon_{\mathrm{bm}}}-\mathscr{D}_{\mathrm{OB}_{\mathrm{p}}} C_{\mathrm{OB}_{\mathrm{p}}} \pi_{\mathrm{r}, \mathrm{T}}, \\
& \frac{\mathrm{d} C_{\mathrm{OB}_{\mathrm{a}}}}{\mathrm{d} t}=\mathscr{D}_{\mathrm{OB}_{\mathrm{p}}} C_{\mathrm{OB}_{\mathrm{p}}} \pi_{\mathrm{r}, \mathrm{T}}-\mathscr{A}_{\mathrm{OB}_{\mathrm{a}}} C_{\mathrm{OB}_{\mathrm{a}}}, \\
& \frac{\mathrm{d} C_{\mathrm{OC}_{\mathrm{a}}}}{\mathrm{d} t}=\mathscr{D}_{\mathrm{OC}_{\mathrm{p}}} C_{\mathrm{OC}_{\mathrm{p}}} \pi_{\mathrm{a}, \mathrm{R}}-\mathscr{A}_{\mathrm{OC}_{\mathrm{a}}} C_{\mathrm{OC}_{\mathrm{a}}} \pi_{\mathrm{a}, \mathrm{T}},
\end{aligned}
$$

for details see $[4,5]$ and references therein. In Eqs. (1) - (3),

- $C_{\mathrm{OB}_{\mathrm{u}}}, C_{\mathrm{OB}_{\mathrm{p}}}, C_{\mathrm{OB}_{\mathrm{a}}}$ denote the concentrations of osteoblast progenitor cells, osteoblast precursor cells, and active osteoblasts, whereas $C_{\mathrm{OC}_{\mathrm{p}}}$ and $C_{\mathrm{OC}_{\mathrm{a}}}$ denote the concentrations of osteoclast precursor cells and active osteoclasts;

- $\mathscr{D}_{\mathrm{OB}_{\mathrm{u}}}, \mathscr{D}_{\mathrm{OB}_{\mathrm{p}}}$, and $\mathscr{D}_{\mathrm{OC}_{\mathrm{p}}}$ denote the differentiation rates of osteoblast progenitor cells, osteoblast precursors, and osteoclast precursors; $\mathscr{P}_{\mathrm{OB}_{\mathrm{p}}}$ denotes the proliferation rate of os- 
teoblast precursor cells; $\mathscr{A}_{\mathrm{OB}_{\mathrm{a}}}$ and $\mathscr{A}_{\mathrm{OC}_{\mathrm{a}}}$ denote the apoptosis rates of active osteoblasts and active osteoclasts;

- $\pi_{\mathrm{a}, \mathrm{T}}$, and $\pi_{\mathrm{r}, \mathrm{T}}$ are the activation and repression functions regulating osteoblast differentiation and osteoclast apoptosis by TGF- $\beta$, whereas $\pi_{\mathrm{a}, \mathrm{R}}$ is the activation function regulating osteoclast differentiation by the RANK-RANKL-OPG system; the nomenclature of these functions, ranging between $\pi=[0,1]$, is to be understood as follows: the first subscript defines whether the function is related to activation (index "a") or repression (index " $r$ ") of a cell transformation (e.g. differentiation) induced by the substance indicated by the second letter in the subscript ("T" represents TGF- $\beta$ and "R" represents the RANK-RANKL-OPG system);

- anabolic mechanoregulation is considered via overuse-related function $\Pi_{\varepsilon_{\mathrm{bm}}}=[0,1]$, controlling proliferation of osteoblast precursor cells as a function of the strain tensor experienced by the extravascular bone matrix, $\varepsilon_{\mathrm{bm}}$; and catabolic mechanoregulation is realized via a disuse-related source term in the expression for the concentration of RANKL; see Section 2.2.4 and [4] for details on the model implementation.

\subsubsection{Quantification of the bone composition}

We assume that bone is always composed of pore space and extravascular bone matrix, i.e. the process of bone mineralization necessitating the consideration of osteoid, the substance laid down by osteoblasts which is transformed to solid bone matrix after a few days, is, for the time being, assumed to be negligible. The bone constituents are quantified by means of volume fractions $f_{i}$, with

$$
f_{\mathrm{vas}}(t)+f_{\mathrm{bm}}(t)=1,
$$

where $f_{\text {vas }}$ is the volume fraction of Haversian pore space, and $f_{\mathrm{bm}}$ is the volume fraction of extravascular bone matrix. The action of active osteoclasts cause increase of the volume fraction of pore space and the action of active osteoblasts cause decrease of the volume fraction of the extravascular bone matrix, reading in mathematical terms

$$
\frac{\mathrm{d} f_{\mathrm{vas}}}{\mathrm{d} t}=k_{\mathrm{res}} C_{\mathrm{OC}_{\mathrm{a}}}-k_{\text {form }} C_{\mathrm{OB}_{\mathrm{a}}}
$$

with $k_{\text {res }}$ as the resorption rate quantifying the amount of bone resorbed by active osteoclasts, and with $k_{\text {form }}$ as the formation rate quantifying the how much bone matrix is formed by active osteoblasts. Resorption and formation rates are considered as constant properties, intrinsic to the respective cells. Analogously, the evolution of the volume fraction of extravascular bone matrix follows from bone resorption-related decrease, and bone formation-related increase. I.e.

$$
\frac{\mathrm{d} f_{\mathrm{bm}}}{\mathrm{d} t}=k_{\mathrm{res}} C_{\mathrm{OC}_{\mathrm{a}}}-k_{\mathrm{form}} C_{\mathrm{OB}_{\mathrm{a}}} .
$$




\subsubsection{Continuum micromechanics-based stiffness homogenization}

Continuum micromechanics provides a physically profound tool for relating the volume fractions of bone to corresponding mechanical properties [26, 27, 14, 15]. Within a representative volume element (RVE) of cortical bone whose characteristic length is chosen such that it is reasonably represented as two-phase material composed of pore space and extravascular bone matrix, the anisotropic macroscopic stiffness tensor governing the elastic deformations reads

$$
\mathbb{C}_{\text {cort }}^{\text {hom }}=\sum_{r} f_{r} \mathbb{C}_{r} \mathbb{A}_{r}^{\text {est }},
$$

with $\mathbb{C}_{r}$ as the microscopic fourth-order stiffness tensors of constituent $r, r=$ vas, bm, and $\mathrm{A}_{r}^{\text {est }}$ as the estimate of the corresponding fourth-order strain concentration tensor, relating the macroscopic and microscopic second-order strain tensors [15]. $\mathbb{A}_{r}^{\text {est }}$ can be estimated based on Eshelby's classical matrix-inclusion problem [29, 30], by means of the Mori-Tanaka scheme $[31,32]$,

$$
\mathbb{A}_{r}^{\text {est }}=\left[\mathbb{I}+\mathbb{P}_{r}^{\mathrm{bm}}:\left(\mathbb{C}_{r}-\mathbb{C}_{\mathrm{bm}}\right)\right]^{-1}:\left\{\sum_{s} f_{s}\left[\mathbb{I}+\mathbb{P}_{s}^{\mathrm{bm}}:\left(\mathbb{C}_{s}-\mathbb{C}_{\mathrm{bm}}\right)\right]^{-1}\right\}^{-1},
$$

where index $r$ denotes either of the three phases, and the summation over index $s$ includes all of them, $s=$ vas, bm. Furthermore, $\mathbb{I}$ is the fourth-order unit tensor with the components defined through the Kronecker delta, $\delta_{i j}=1$ for $i=j$ and zero otherwise, as $I_{i j k l}=1 / 2\left(\delta_{i k} \delta_{j l}+\delta_{i l} \delta_{j k}\right)$, and $\mathbb{P}_{r}^{\mathrm{bm}}$ is the fourth-order Hill-tensor of phase $r$ embedded in a matrix with stiffness $\mathbb{C}_{\mathrm{bm}}$. For a detailed explanation how the Hill tensor of a cylindrical phase (such as Haversian pore space) is calculated, see [33, 34]. Numerical evaluation of Eqs. (7) and (8) requires knowledge of phase volume fractions and phase stiffness tensors. The volume fractions follow from the bone cell population model, see Section 2.2.2, whereas the phase stiffness tensors are chosen in the line of [17], with the Haversian pore space assumed as sealed and filled with water-like fluid (from a mechanical point of view), and the stiffness tensor of extravascular bone matrix being based on the ultrasonics tests by [35], see [17] for details.

\subsubsection{Establishment of mechanoregulatory feedback mechanisms}

We are left with definition of the mechanoregulatory functions $\Pi_{\varepsilon_{\varepsilon_{b m}}}$ and $P_{\mathrm{RL}, \varepsilon_{\mathrm{bm}}}$. To this end, we assume that both $\Pi_{\varepsilon_{\mathrm{bm}}}$ and $P_{\mathrm{RL}, \varepsilon_{\mathrm{bm}}}$ are directly controlled by the strains experienced by the extravascular bone matrix, $\varepsilon_{\mathrm{bm}}$. The macroscopic stress tensor acting on cortical bone, $\Sigma_{\text {cort }}$, is related to the corresponding macroscopic strain tensor, $\mathbf{E}_{\text {cort }}$, via the macroscopic stiffness tensor obtained from Eq. (7), through a linear elastic constitutive law,

$$
\mathbf{E}_{\text {cort }}=\left(\mathbb{C}_{\text {cort }}^{\text {hom }}\right)^{-1}: \Sigma_{\text {cort }} .
$$

As pointed out earlier the strain in the extravascular bone matrix affects the activity of the osteocytes embedded in that matrix. Making use of the microelastic model presented in the 
previous section, $\varepsilon_{\text {bm }}$ can be mathematically related to $\mathbf{E}_{\text {cort }}$, and, via Eq. (9) to a macroscopic stress tensor, through

$$
\varepsilon_{\mathrm{bm}}=\mathbb{A}_{\mathrm{bm}}^{\mathrm{est}}: \mathbf{E}_{\mathrm{cort}} .
$$

From a practical point of view, it is advantageous to express the three-dimensional (3D) strain state represented by $\varepsilon_{\mathrm{bm}}$ by a corresponding scalar quantity. From the several quantities suggested in conventional approaches $[36,37,38]$, we choose the strain energy density (SED) as mechanoregulatory quantity. However, contrary to conventional approaches, we consider the SED on the observation scale of extravascular bone matrix (where mechanosensing actually takes place), rather than on the macroscopic observation scale of cortical bone. The microscopic SED, experienced by the extravascular bone matrix reads as

$$
w_{\varepsilon_{\mathrm{bm}}}=\frac{1}{2} \varepsilon_{\mathrm{bm}}: \mathbb{C}_{\mathrm{bm}}: \varepsilon_{\mathrm{bm}} .
$$

Not only the strain magnitude, but also other characteristics of the mechanical loading exerted onto bone have been identified as governing factors of mechanical regulation, e.g. the rate of the experienced strains, or the frequency of the applied loading [25, 39]. Strain rate and loading frequency are subsequently considered as constant, thus the strain magnitude is the only mechanoregulatory quantity, governing both catabolic and anabolic bone remodeling responses.

The anabolic mechanoregulatory function, $\Pi_{\varepsilon_{\mathrm{bm}}}$, is defined as

$$
\Pi_{\varepsilon_{\mathrm{bm}}}=\Pi_{\varepsilon_{\mathrm{bm}, \mathrm{st}}}\left[1+\lambda\left(\frac{w_{\varepsilon_{\mathrm{bm}}}}{w_{\varepsilon_{\mathrm{bm}, \mathrm{st}}}}-1\right)\right],
$$

with $w_{\varepsilon_{\mathrm{bm}, \mathrm{st}}}$ as set point SED corresponding to balanced bone turnover at steady-state, $\Pi_{\varepsilon_{\mathrm{bm}, \mathrm{st}}}$ denoting the steady-state value of $\Pi_{\varepsilon_{\mathrm{bm}}}$, and $\lambda$ as parameter allowing for adjustment of the proliferation rate. The bilinear function $\Pi_{\varepsilon_{\mathrm{bm}}}$ is defined by $\lambda=0$ for $w_{\varepsilon_{\mathrm{bm}}} \leq w_{\varepsilon_{\mathrm{bm}, \mathrm{st}}}$ and $\lambda>0$ otherwise. Mechanically induced production of RANKL, $P_{\mathrm{RL}, \varepsilon_{\mathrm{bm}}}$, is assumed to be only initiated by mechanical disuse, implying increased bone resorption. $P_{\mathrm{RL}, \varepsilon_{\mathrm{bm}}}$ is defined analogously to $\Pi_{\varepsilon_{\mathrm{bm}}}$, and reads as

$$
P_{\mathrm{RL}, \varepsilon_{\mathrm{bm}}}=\kappa\left(1-\frac{w_{\varepsilon_{\mathrm{bm}}}}{w_{\varepsilon_{\mathrm{bm}, \mathrm{st}}}}\right)
$$

with $\kappa$ as a parameter allowing for adjustment of mechanically induced source of RANKL. The bilinear function for $P_{\mathrm{RL}, \varepsilon_{\mathrm{bm}}}$ is defined by $\kappa=0$ for $w_{\varepsilon_{\mathrm{bm}}} \geq w_{\varepsilon_{\mathrm{bm}, \mathrm{st}}}$ and $\kappa>0$ otherwise. For introduction of $P_{\mathrm{RL}, \varepsilon_{\mathrm{bm}}}$ in the mathematical framework given by Eqs. (11) - (13) see [5].

\section{Numerical studies: Mechanical disuse regimes}

In the subsequently presented numerical study, the general model response to mechanical disuse and reuse is investigated. The corresponding simulations are based on the following 
parameters: Ratio $a_{\mathscr{D}_{\mathrm{B}} \mathrm{u}}$, defining the ratio of osteoblast precursor proliferation to differentiation at steady state, is set to $a_{\mathscr{D}_{\mathrm{B}}}=0.1$ (i.e. at steady state $10 \%$ of the overall supply of new osteoblast precursors, governed by Eq. (1), stems from proliferation); parameters $\kappa$ and $\lambda$, implemented for calibration of the mechanoresponsiveness in Eqs. (12) and (13) are set to $\kappa=10^{5} \mathrm{pM} /$ day and $\lambda=1.25$, while $\Pi_{\varepsilon_{\text {bm st }}}=0.5$; the vascular porosity at steady-state conditions is set to $f_{\text {vas,st }}=0.05$; the normal loading (although physiological loading scenarios are expected to be of three-dimensional nature we consider, for the sake of simplicity, uniaxial loading) is specified by $\Sigma_{\text {cort }, i j}^{\text {normal }}=-30 \mathrm{MPa}$ if $i j=33$ and zero otherwise, and a disuse loading regime is simulated with $\sum_{\text {cort }, 33}^{\text {disuse }}=-25 \mathrm{MPa}$ for $0 \leq t \leq 2000 \mathrm{~d}$ (after disuse the loading is set back to $\sum_{\text {cort }, 33}^{\text {normal }}$ and the system is observed for further $3000 \mathrm{~d}$ ); all other parameters are chosen as calibrated in $[4,5]$ and are, for the sake of conciseness not further elaborated in this paper.

Evaluation of the coupled methodology presented in Section 2 reveals, on the basis of above defined parameters, a sudden drop of the experienced microscopic SED, corresponding to the imposed disuse scenario, see Fig. 1(a). This deviation from steady-state loading conditions $\left(w_{\varepsilon_{\mathrm{bm}}}<w_{\varepsilon_{\mathrm{bm}, \mathrm{s}}}\right)$ provokes increased production of RANKL, governed by Eq. (13), leading to an increase of the concentration of active osteoclasts (relative to the concentration of active osteoblasts), thus $\left(C_{\mathrm{OC}_{\mathrm{a}}} / C_{\mathrm{OC}_{\mathrm{a}}, \mathrm{st}}\right)>\left(C_{\mathrm{OB}_{\mathrm{a}}} / C_{\mathrm{OB}_{\mathrm{a}}, \mathrm{st}}\right)$, see Fig. 1(b). Obviously, this alteration of the cell concentrations entails increased bone resorption, and consequently an increase of the volume fraction of vascular pore space through Eq. (5), see Fig. 1(c). An increasing porosity of the RVE is accompanied by a corresponding weakening of the RVE, i.e. the macroscopic stiffness increases, and given that the prescribed macroscopic loading is assumed to be constant, the deformations increase, and so does the microscopic SED, as governed by Eqs. (11) and (12). Over time, the coupled model converges to a new, disuse-related steady-state, with equilibrated bone turnover. This is observed after $\approx 1000 \mathrm{~d}$, indicated by a subsequently constant volume fraction of vascular pore space, see the black graph in Fig. 1(c), by an equilibrated SED $\left(w_{\varepsilon_{\mathrm{bm}}}=w_{\varepsilon_{\text {bm.st }}}\right)$, see Fig. 1(a), which results in setting the disuserelated additional production of RANKL back to zero. Consequently, the cell concentrations return to the original level relating to the steady-state $\left(C_{i} / C_{i, \text { st }}=1\right)$, see Fig. 1(b).

Returning to the original macroscopic load at $t=2000 \mathrm{~d}, \Sigma_{\text {cort }, 33}=\Sigma_{\text {cort }, 33}^{\text {normal implies a }}$ sudden increase of the deformation of the RVE, due to which the proliferation of osteoblast precursors is increased via Eq. (12). Increased osteoblast proliferation is maintained until the original steady-state is reached (occurs at $t \gg 5000$ days). The results indicated by the black graphs in Fig. 1 clearly resemble, at least qualitatively, the mechanoregulatory behavior observed experimentally for bone subjected to disuse scenarios, compare e.g. the investigations of Vico and co-workers during and after space flight [40, 41]. In essence, exposure to microgravity, accompanied by reduced loading to which bone is subjected, leads to adaption of the bone mass towards a new equilibrium after a certain period of time. When subjected again to terrestrial gravity, bone responses by recovering, i.e. by adjusting its mass to the original level. This behavior, as interpreted in terms of corresponding volume fractions of the bone constituents, is predicted by the above described simulation, see Fig. 1 . 
(a)

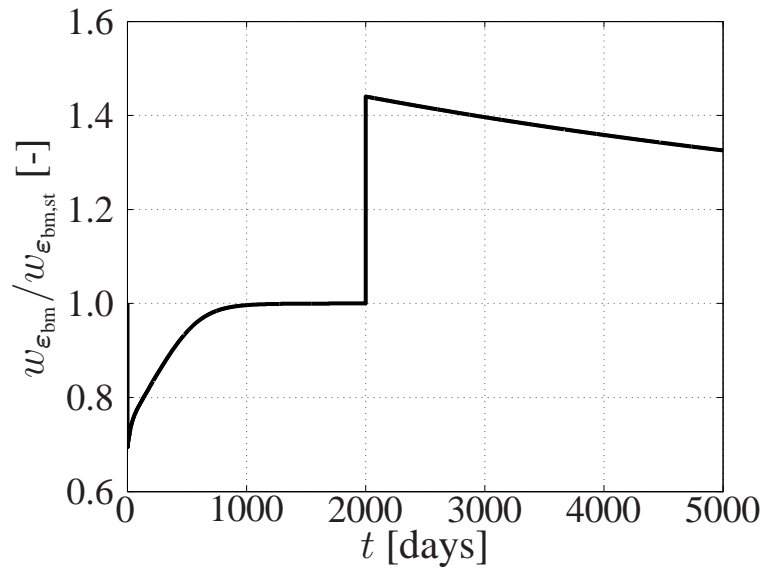

(b)

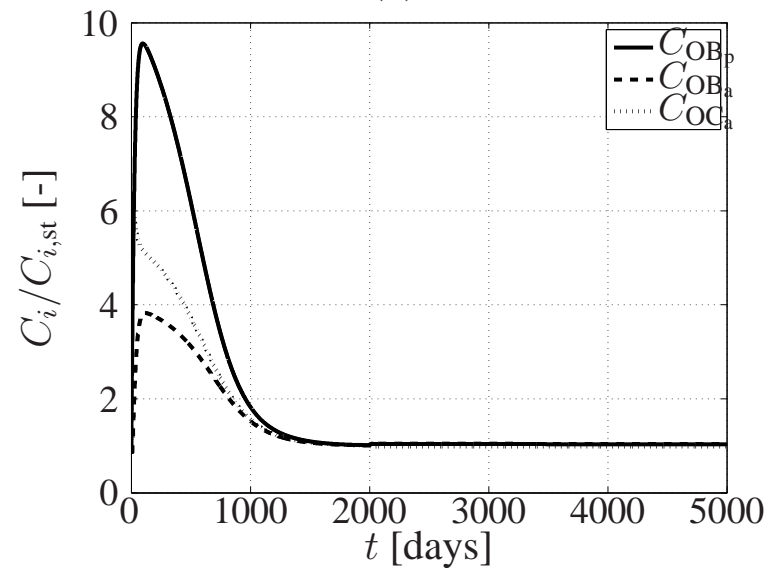

(c)

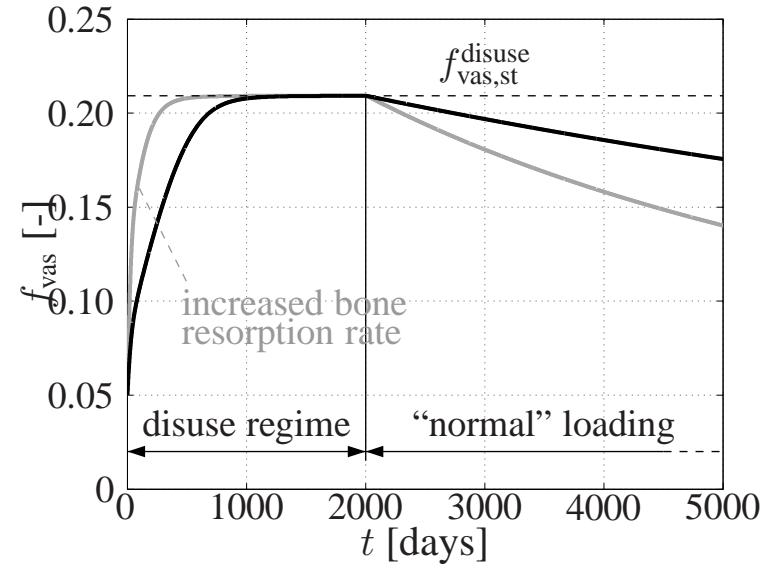

Figure 1. Numerical results for a disuse-scenario, characterized by $\Sigma_{\text {cort }, 33}=\Sigma_{\text {cort }, 33}^{\text {disuse }}=$ $-25 \mathrm{MPa}$ for $0 \leq t \leq 2000 \mathrm{~d}$, and by $\Sigma_{\text {cort }, 33}=\Sigma_{\text {cort }, 33}^{\text {normal }}=-30 \mathrm{MPa}$ for $t>2000 \mathrm{~d}$ : evolutions of (a) microscopic SED $w_{\varepsilon_{\mathrm{bm}}}$, normalized with respect to $w_{\varepsilon_{\mathrm{bm}, \mathrm{st}}}$, (b) bone cell concentrations $C_{i}$, normalized with respect to the steady-state cell concentrations $C_{i, \mathrm{st}}$, and (c) vascular porosity $f_{\text {vas }}$ for $k_{\text {res }}=200 \mathrm{pM}^{-1}$ day $^{-1}$ (black graph) and $k_{\text {res }}=500 \mathrm{pM}^{-1}$ day $^{-1}$ (grey graph) 
The results, as compared with experimental results, furthermore underline in striking fashion that species-dependent calibration of the model is crucial. E.g. measurements on the bone mineral density (BMD) carried out on cosmonauts after a six month-exposure to microgravity [41] show that the BMD of cortical bone decreases by not more than $2.5 \%$ (in the distal radius) and by $4.3 \%$ (in the distal tibia), respectively. In other words, the average rate of bone loss amounted to $0.42 \%$ /month (distal radius) and $0.72 \% /$ month (distal tibia), respectively. The bone loss rate revealed by the computational simulations, $0.48 \% / \mathrm{month}$, see the black graph in Fig. 1(c), fits well to the experimentally obtained range. Investigating the disuse behavior of other species, quite different numbers are obtained - e.g. rats show much higher rates of bone loss as exposed to microgravity. Several explanations are conceivable, e.g. that the resorption activity of active osteoblasts in rats is increased compared to humans. To study the relevance of this explanation, a second disuse-study is carried out, with the resorption rate being multiplied by factor 2.5 , i.e. $k_{\text {res }}=500(\mathrm{pM} \text { day })^{-1}$. The grey graph in Fig. 1(c) shows that increasing the resorption rate leads to distinctive acceleration of bone resorption, i.e. adaption to the new loading regime is concluded much earlier. I.e. the response of the presented model can be adjusted to different species (but also to different bone tissues), by reasonable adjustment of the underlying parameters.

\section{Conclusions}

For the first time, the model proposed here provides biophysically reasonable estimates of the stiffness changes in cortical bone, governed by both biochemical and biomechanics factors. This was achieved through coupling of a bone cell population model with a microelastic model of bone stiffness, through a feedback loop implemented on the basis of the microscopic strain energy density which is experienced on the observation scale of extravascular bone matrix, controlling both anabolic and catabolic bone remodeling responses.

For demonstration of the capability of the approach, a mechanical loading regime comprising mechanical disuse and reuse (as experienced in spaceflight scenarios). It was revealed that the model soundly reproduces, at least qualitatively, physiologically observed features, such as fast loss of bone because of unloading, and slow increase of bone mass after exposing the bone to normal mechanical loading. These outcomes of the numerical study presented in the previous section show that the proposed approach captures important features of mechanoregulation of bone remodeling.

To improve the model, in future studies, we will focus on consideration of mechanoregulation in mechanistic fashion, through explicit introduction of the actions of osteocytes and the signaling pathways by which osteocytes are able to direct osteoclasts and osteoblasts. This will allow us to further validate the model and eventually utilize it as interpretative and predictive tool. 


\section{Acknowledgment}

Financial support by the Australian Research Council (ARC), in the framework of the project Multi-scale modeling of transport through deformable porous materials (project number DP0988427), and by the European Research Council (ERC), in the framework of the project Multiscale poro-micromechanics of bone materials, with links to biology and medicine (project number FP7-257023), is gratefully acknowledged.

\section{References}

[1] J.E. Aubin. Bone stem cells. Journal of Cellular Biochemistry, Supplements 30/31:7382, 1998.

[2] R.B. Martin, D.B. Burr, and N.A. Sharkey. Skeletal Tissue Mechanics. Springer Verlag, 1998.

[3] V. Lemaire, F.L. Tobin, L.D. Greller, C.R. Cho, and L.J. Suva. Modeling of the interactions between osteoblast and osteoclast activities in bone remodeling. Journal of Theoretical Biology, 229(3):293-309, 2004.

[4] P. Pivonka, J. Zimak, D.W. Smith, B.S. Gardiner, C.R. Dunstan, N.A. Sims, T.J. Martin, and G.R. Mundy. Model structure and control of bone remodeling: A theoretical study. Bone, 43(2):249-263, 2008.

[5] P. Pivonka, J. Zimak, D.W. Smith, B.S. Gardiner, C.R. Dunstan, N.A. Sims, T.J. Martin, and G.R. Mundy. Theoretical investigation of the role of the RANK-RANKL-OPG system in bone remodeling. Journal of Theoretical Biology, 262(2):306-316, 2010.

[6] C.T. Rubin and L.E. Lanyon. Regulation of bone mass by mechanical strain magnitude. Calcified Tissue International, 37(4):411-417, 1985.

[7] C.H. Turner and F.M. Pavalko. Mechanotransduction and functional response of the skeleton to physical stress: The mechanisms and mechanics of bone adaptation. Journal of Orthopaedic Science, 3(6):346-355, 1998.

[8] R.B. Martin. Toward a unifying theory of bone remodeling. Bone, 26(1):1-6, 2000.

[9] L. Geris, J. Vander Sloten, and H. Van Oosterwyck. In silico biology of bone modeling and remodeling: regeneration. Philosophical Transactions of the Royal Society London A, 367(1895):2031-2053, 2009.

[10] L.F. Bonewald and M.L. Johnson. Osteocytes, mechanosensing and Wnt signaling. Bone, 42(4):606-615, 2008.

[11] C.H. Turner and A.G. Robling. Mechanisms by which exercise improves bone strength. Journal of Bone and Mineral Metabolism, 23(S1):16-22, 2005. 
[12] J.-Y. Rho, L. Kuhn-Spearing, and P. Zioupos. Mechanical properties and the hierarchical structure of bone. Medical Engineering and Physics, 20(2):92-102, 1998.

[13] S. Weiner and H.D. Wagner. The material bone: Structure-mechanical function relations. Annual Review of Materials Science, 28(1):271-298, 1998.

[14] A. Zaoui. Structural Morphology and Constitutive Behavior of Microheterogeneous Materials, chapter 6, pages 291 - 347. Springer-Verlag, Wien New York, 1997. In [28].

[15] A. Zaoui. Continuum micromechanics: survey. Journal of Engineering Mechanics (ASCE), 128(8):808-816, 2002.

[16] Ch. Hellmich and F.-J. Ulm. Micromechanical model for ultra-structural stiffness of mineralized tissues. Journal of Engineering Mechanics (ASCE), 128(8):898 - 908, 2002.

[17] A. Fritsch and C. Hellmich. 'Universal' microstructural patterns in cortical and trabecular, extracellular and extravascular bone materials: micromechanics-based prediction of anisotropic elasticity. Journal of Theoretical Biology, 244(4):597-620, 2007.

[18] J. Vuong and C. Hellmich. Bone fibrillogenesis and mineralization: Quantitative analysis and implications for tissue elasticity. Journal of Theoretical Biology, 287:115 - 130, 2011.

[19] Ch. Hellmich, J.-F. Barthélémy, and L. Dormieux. Mineral-collagen interactions in elasticity of bone ultrastructure - a continuum micromechanics approach. European Journal of Mechanics - A/Solids, 23(5):783 - 810, 2004.

[20] E. Seeman. Invited review: pathogenesis of osteoporosis. Journal of Applied Physiology, 95(5):2142-2151, 2003.

[21] D.M.L. Cooper, C.D.L. Thomas, J.G. Clement, A.L. Turinsky, C.W. Sensen, and B. Hallgrímson. Age-dependent change in the 3D structure of cortical porosity at the human femoral midshaft. Bone, 40(4):957-965, 2007.

[22] L.F. Bonewald and S.L. Dallas. Role of active and latent transforming growth factor- $\beta$ in bone formation. Journal of Cellular Biochemistry, 55(3):350-357, 1994.

[23] K. Janssens, P. ten Dijke, S. Janssens, and W. Van Hul. Transforming growth factor- $\beta 1$ to the bone. Endocrine Reviews, 26(6):743-774, 2005.

[24] L.C. Hofbauer, C.A. Kühne, and V. Viereck. The OPG/RANKL/RANK system in metabolic bone diseases. Journal of Musculoskeletal Neuronal Interactions, 4(3):268$275,2004$.

[25] A.G. Robling, A. Castillo, and C.H. Turner. Biomechanical and molecular regulation of bone remodeling. Annual Review of Biomedical Engineering, 8:455-498, 2006. 
[26] R. Hill. Elastic properties of reinforced solids: some theoretical principles. Journal of Mechanics and Physics of Solids, 11(5):357-372, 1963.

[27] R. Hill. Continuum micro-mechanics of elastoplastic polycrystals. Journal of Mechanics and Physics of Solids, 13(2):89-101, 1965.

[28] P.M. Suquet. Continuum Micromechanics, volume 377 of CISM Courses and Lectures. Springer Verlag, Wien New York, 1997.

[29] J. Eshelby. The determination of the elastic field of an ellipsoidal inclusion, and related problems. Proceedings of the Royal Society London, Series A, 241:376 - 396, 1957.

[30] N. Laws. The determination of stress and strain concentrations at an ellipsoidal inclusion in an anisotropic material. Journal of Elasticity, 7(1):91 - 97, 1977.

[31] T. Mori and K. Tanaka. Average stress in matrix and average elastic energy of materials with misfitting inclusions. Acta Metallurgica, 21(5):571 - 574, 1973.

[32] Y. Benveniste. A new approach to the application of Mori-Tanaka's theory in composite materials. Mechanics of Materials, 6:147 - 157, 1987.

[33] A. Fritsch, L. Dormieux, and Ch. Hellmich. Porous polycrystals built up by uniformly and axisymmetrically oriented needles: homogenization of elastic properties. Comptes Rendus Mécanique, 334(3):151 - 157, 2006.

[34] C. Hellmich, C. Kober, and B. Erdmann. Micromechanics-based conversion of CT data into anisotropic elasticity tensors, applied to FE simulations of a mandible. Annals of Biomedical Engineering, 36(1):108-122, 2008.

[35] R.B. Ashman, S.C. Cowin, W.C. Van Buskirk, and J.C. Rice. A continuous wave technique for the measurement of the elastic properties of cortical bone. Journal of Biomechanics, 17(5):349-361, 1984.

[36] D.P. Fyhrie and D.R. Carter. A unifying principle relating stress to trabecular bone morphology. Journal of Orthopaedic Research, 4(3):304-317, 1986.

[37] R. Huiskes, H. Weinans, H.J. Grootenboer, M. Dalstra, B. Fudala, and T.J. Slooff. Adaptive bone-remodeling theory applied to prosthetic-design analysis. Journal of Biomechanics, 20(11-12):1135-1150, 1987.

[38] J.M. Garcia-Aznar, J.H. Kuiper, M.J. Gómez-Benito, M. Doblaré, and J.B. Richardson. Computational simulation of fracture healing: Influence of interfragmentary movement on the callus growth. Journal of Biomechanics, 40(7):1467-1476, 2007.

[39] E. Ozcivici, Y.K. Luu, B. Adler, Y.-X. Qin, J. Rubin, S. Judex, and C.T. Rubin. Mechanical signals as anabolic agents in bone. Nature Reviews Rheumatology, 6(1):50-59, 2010 . 
[40] L. Vico and C. Alexandre. Microgravity and bone adaption at the tissue level. Journal of Bone and Mineral Research, 7(S2):445-447, 1992.

[41] L. Vico, P. Collet, A. Guignandon, M.-H. Lafage-Proust, T. Thomas, M. Rehailia, and C. Alexandre. Effects of long-term microgravity exposure on cancellous and cortical weight-bearing bones of cosmonauts. The Lancet, 355(9215):1607-1611, 2000. 